\title{
Escleroterapia com espuma de polidocanol em veias safenas magnas e suas tributárias bilateralmente em tempo único
}

\author{
Bilateral foam polidocanol sclerotherapy of great saphenous veins and their tributaries \\ in synchronous procedure
}

\author{
Luiz Antonio Miranda ${ }^{1}$ (D), Rachel Cristina do Carmo $^{2}$ (D), Cláudia Carvalho Sathler-Melo ${ }^{3}$ (D), \\ Guilherme de Castro-Santos ${ }^{4}$ iD
}

\begin{abstract}
Resumo
Contexto: A insuficiência venosa crônica é uma entidade com alta prevalência. Os casos avançados apresentam morbidade elevada. Objetivos: Avaliar os riscos e benefícios da escleroterapia com espuma de polidocanol em pacientes que foram submetidos ao tratamento das veias safenas magnas bilateralmente em tempo único. Métodos: Foram revistos retrospectivamente 55 pacientes (110 membros) portadores de incompetência bilateral das veias safenas magnas submetidas a tratamento escleroterápico com espuma bilateralmente, em tempo único, usando uma dose máxima de $20 \mathrm{~mL}$ de espuma de polidocanol por paciente. Resultados: Das 110 safenas analisadas, obteve-se a oclusão de 81 (73,6\%) com uma sessão, de 106 (96,3\%) com duas sessões e de 110 (100\%) com três sessões. Houve oclusão bilateral das safenas magnas em 27 pacientes (50\%) em uma sessão, em 34 (62\%) em duas sessões e em 55 (100\%) em três sessões. De 11 pacientes portadores de úlceras, houve cicatrização total de sete (63\%) e parcial de três (27\%) 42 dias após a escleroterapia. Houve lipotimia autolimitada e escotomas visuais em um paciente (1,8\%) e manchas em três (5,45\%); 19 pacientes (34,5\%) foram submetidos a punção para drenagem de coágulo retido. Conclusões: A escleroterapia com espuma de polidocanol em veias safenas magnas em tempo único mostrou-se uma técnica segura e eficaz em pacientes selecionados.
\end{abstract}

Palavras-chave: escleroterapia com espuma; varizes bilaterais; complicações.

\begin{abstract}
Background: Chronic venous insufficiency is a highly prevalent disease. Advanced cases have high morbidity. Objectives: To evaluate the risks and benefits of foam sclerotherapy in patients who underwent bilateral treatment of the great saphenous veins in a single procedure, in selected cases of advanced venous insufficiency. Methods: We retrospectively reviewed 55 patients (110 limbs) with bilateral incompetence of the great saphenous veins who had undergone foam sclerotherapy treatment bilaterally, using a maximum dose of $20 \mathrm{ml}$ of foam per patient and inelastic compression. Results: In 81 (73.6\%) of the 110 saphenous veins analyzed, occlusion was obtained in the first session. After a second session this figure rose to 106 (96.3\%) and all 110 (100\%) veins were occluded after three sessions. Bilateral occlusion of the great saphenous veins was achieved in 27 patients (50\%) in one session, in 34 (62\%) patients in two sessions, and in 55 (100\%) patients in three sessions. At 42 days after sclerotherapy, there was complete ulcer healing in seven (63\%) of the 11 patients with ulcers and partial healing in 3 (27\%) of these patients. One patient (1.8\%) had self-limited lipothymia and visual scotomas, 3 patients (5.45\%) had skin spots, and 19 patients (34.5\%) developed retained intravascular coagulum. Conclusions: Bilateral foam sclerotherapy in a synchronous procedure is an option to be considered for treatment of varicose veins of the lower limbs.
\end{abstract}

Keywords: foam sclerotherapy; bilateral varicose veins; complications.

Como citar: Miranda LA, Carmo RC, Sathler-Melo CC, Castro-Santos G. Escleroterapia com espuma de polidocanol em veias safenas magnas e suas tributárias bilateralmente em tempo único. J Vasc Bras. 2021;20:e20200178. https:// doi.org/10.1590/1677-5449.200178

${ }^{1}$ Hospital Infantil Padre Anchieta, Belo Horizonte, MG, Brasil.

${ }^{2}$ Hospital Militar de Área de Manaus - HMAM, Manaus, AM, Brasil.

${ }^{3}$ Clínica Angiovasc, Nova Lima, MG, Brasil.

${ }^{4}$ Universidade Federal de Minas Gerais - UFMG, Belo Horizonte, MG, Brasil.

Fonte de financiamento: Nenhuma.

Conflito de interesse: Os autores declararam não haver conflitos de interesse que precisam ser informados.

Submetido em: Setembro 30, 2020. Aceito em: Janeiro 18, 2021.

O estudo foi realizado no Hospital Infantil Padre Anchieta, Belo Horizonte, MG, Brasil.

Copyright $(\mathrm{C} 2021$ Os autores. Este é um artigo publicado em acesso aberto (Open Access) sob a licença Creative Commons Attribution, que permite uso, distribuição e reprodução em qualquer meio, sem restrições desde que o trabalho original seja corretamente citado. 


\section{INTRODUÇÃO}

As varizes dos membros inferiores são conhecidas desde a antiguidade, quando foram descritas pelos egípcios como "serpentinas" enroladas nas pernas. No século V a.C., Hipócrates as comparava com "cachos de uvas" e sugeria que ferro incandescente poderia curá-las. Em 1882, Tredenlegurger, realizando o striping das veias, lançou as bases para o tratamento cirúrgico que persiste até os dias atuais ${ }^{1}$.

Até o século passado, o padrão-ouro do tratamento das varizes dos membros inferiores era a cirurgia convencional. Evoluímos para as cirurgias minimamente invasivas com a introdução da agulha de crochê (1974), abrasão por endolaser e radiofrequência e escleroterapia por espuma².

A primeira publicação sobre escleroterapia com espuma foi feita por Orbach, em $1950^{3}$. Cabrera, Monfreux, Tessari etal. são os responsáveis pelos avanços atuais ${ }^{4,5}$. Lunkeinheimer, em 1963, foi o primeiro a usar o polidocanol, substância esclerosante mais usada para o tratamento da insuficiência venosa crônica ${ }^{6}$. O polidocanol pode ser aplicado como esclerosante na forma líquida ou como espuma, sendo que a escleroterapia com espuma resultou maior eficácia ${ }^{7}$. O uso do esclerosante na forma de microespuma, ao deslocar o sangue no interior do vaso, minimiza a diluição do mesmo e facilita o reconhecimento de sua concentração intravenosa, o que não ocorre na forma líquida ${ }^{7}$. Associa-se à reação alérgica em 3:1.000 aplicações ${ }^{8}$. Em 2003, a revista Archives of Dermatology publicou um editorial intitulado "Foam sclerotherapy: a new era", que, em conjunto com os avanços da ultrassonografia, anteviu o futuro promissor, sem volta, da escleroterapia com espuma?

Uma abordagem bilateral na escleroterapia com espuma consiste em tratar os dois membros em um tempo único. Em tese, a redução do número de sessões e o alívio dos sintomas da insuficiência venosa crônica seriam hipóteses para a sua realização. Na literatura, são escassos os trabalhos avaliando seus riscos e benefícios.

O objetivo deste artigo é descrever a técnica, os resultados e as complicações da escleroterapia bilateral das veias safenas magnas com espuma de polidocanol.

\section{MATERIAIS E MÉTODOS}

No período compreendido entre dezembro de 2019 e março de 2020, recebemos 108 pacientes portadores de insuficiência venosa crônica avançada, encaminhados para tratamento cirúrgico e/ou escleroterapia com espuma pelo Sistema Único de Saúde no Hospital Infantil Padre Anchieta, em Belo Horizonte, no estado de Minas Gerais. Desses pacientes, 55 apresentavam refluxo bilateral ostial e/ou segmentar das veias safenas magnas e tiveram os dois membros tratados em tempo

único e avaliados retrospectivamente (Figura 1). Este trabalho foi avaliado e aprovado pelo Comitê de Ética (parecer número 4.245.303).

Foi realizada uma punção ecoguiada (Figura 2A), usando uma agulha 30/8 acoplada a um extensor, ao nível da coxa. O transdutor foi colocado com o canto direito sobre a veia safena magna e angulado a cerca de 45 graus para visualização da penetração perpendicular da agulha. Foram injetados $10 \mathrm{~mL}$ de espuma em cada membro, sendo $5 \mathrm{~mL}$ de polidocanol a $3 \%$ em veia safena magna e $5 \mathrm{~mL}$ de polidocanol a $1 \%$ em tributárias ipsilaterais da veia safena magna, totalizando $20 \mathrm{~mL}$ de espuma em cada paciente.

Para compressão dos membros, após a injeção da espuma, utilizaram-se ataduras de crepom de $15 \mathrm{~cm}$. A técnica de enfaixamento foi de camadas sobrepostas em oito (Figura 2B). As orientações aos pacientes foram as seguintes: retirar as faixas 24 horas após o procedimento; utilizar as meias elásticas durante o dia ou voltar a enfaixar os membros; deambular e manter as atividades habituais. Foram agendados retornos a cada 2 semanas desde o procedimento. Nesse retorno, verificava-se a compressibilidade e a presença ou ausência de fluxo nas veias varicosas ao ultrassom. As que estavam pérvias receberam nova injeção de espuma. Os coágulos retidos foram diagnosticados considerando dolorimento e sensibilidade no trajeto venoso associado à imagem ecogênica intraluminal ao ultrassom vascular com Doppler. Em caso de coágulo retido, foi realizada punção com agulha e drenagem percutânea.

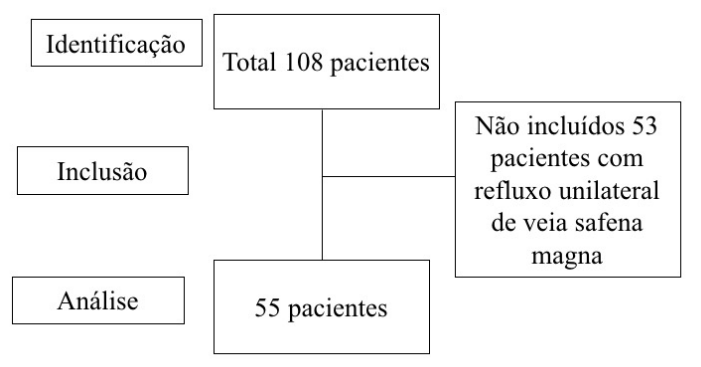

Figura 1. Diagrama de fluxo dos pacientes em estudo.

A

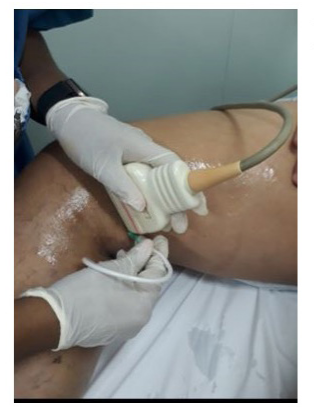

Figura 2. (A) Punção ecoguiada e (B) enfaixamento compressivo. 
O cálculo do tamanho da amostra foi realizado conforme a fórmula: $\mathrm{n}=[\mathrm{Px}(1-\mathrm{P})] /[\mathrm{EP}] 2^{10}$, em que $\mathrm{n}$ é o tamanho da amostra, P é a proporção de ocorrência do evento estudado e $\mathrm{EP}$, o erro padrão. Considerando o trabalho de Bhogal et al. ${ }^{11}$, observamos uma taxa de oclusão das veias safenas em tempo único de $81 \%$ em 2 semanas. Considerando $\mathrm{P}=0,81$ e um erro padrão de 4\%, teríamos um mínimo de 94 membros.

Os dados foram apresentados como média ( \pm desvio padrão ou erro padrão da média) e números. Foi realizado o teste de Shapiro-Wilk para avaliação da normalidade. Variáveis não categóricas, como diâmetros venoso e média da classificação clínica, etiológica, anatômica e patológica (CEAP), foram avaliadas através do teste $t$ de Student. Variáveis categóricas, como presença ou não de oclusão das veias safenas em diversos grupos, foram estudadas através do teste do qui-quadrado com a correção de Yates ou do teste de Fischer quando apropriado. Um valor de $p<0,05$ foi considerado estatisticamente significativo. Toda a análise estatística foi realizada através do software Prism 8 para iOS versão 8.0.1 (GraphPad Software Inc, San Diego, Califórnia).

\section{RESULTADOS}

Ao total, 55 pacientes, 39 do sexo feminino e 16 do sexo masculino, fizeram parte da amostra. $\mathrm{O}$ mais jovem possuía 36 anos e o mais idoso possuía 68 anos (média de 50,7 anos). A média do diâmetro das safenas magnas medidas no terço médio da coxa direita e da coxa esquerda foi de $6,2 \mathrm{~mm}$ (Tabela 1). A CEAP do membro com insuficiência venosa mais avançada: $\mathrm{C} 2$, 7 pacientes; $\mathrm{C} 3,10$ pacientes; $\mathrm{C} 4,22$ pacientes; $\mathrm{C} 5$, 5 pacientes; e C6, 11 pacientes (Tabela 2).

Das 110 safenas analisadas, obteve-se a oclusão de $81(73,6 \%)$ com uma sessão, de $106(96,3 \%)$ com duas sessões e de 110 (100\%) com três sessões. Houve oclusão bilateral das safenas magnas em 27 pacientes $(50 \%)$ em uma sessão, em 34 (62\%) em duas sessões e em 55 (100\%) em três sessões (Figuras 3 e 4).

Não foi observada nenhuma ocorrência de trombose venosa profunda. De 11 pacientes portadores de úlceras, houve cicatrização total em 7 (63\%) e parcial em 3 (27\%) 42 dias após a escleroterapia. Houve lipotimia autolimitada e escotomas visuais em 1 paciente $(1,8 \%)$ e manchas em $3(5,45 \%)$; 19 pacientes $(34,5 \%)$ desenvolveram coágulo retido.

Dos pacientes que desenvolveram coágulo retido, $12(63 \%)$ necessitaram de punção para drenagem em uma ocasião, 9 (47\%) necessitaram de punções em duas ocasiões (Tabela 3). A classificação CEAP em cada grupo a depender do número de ocasiões em que se necessitou de punção e drenagem de coágulo retido está demonstrada na Tabela 4.

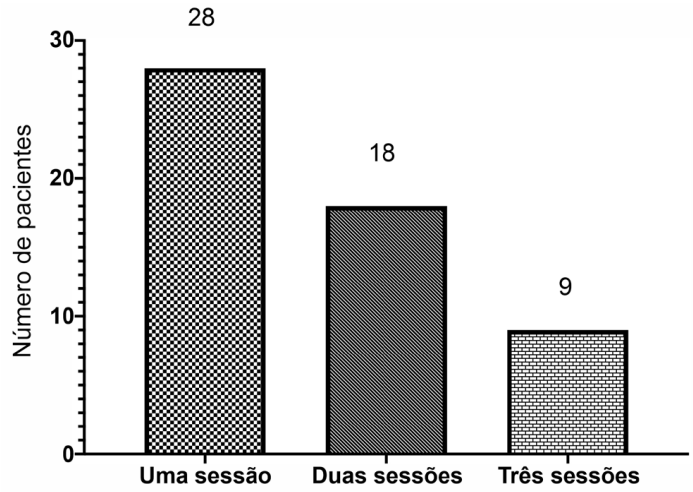

Figura 3. Número de pacientes que necessitaram de uma sessão, duas sessões ou três sessões de escleroterapia com espuma de polidocanol para obter $100 \%$ de oclusão bilateral das veias safenas magnas.

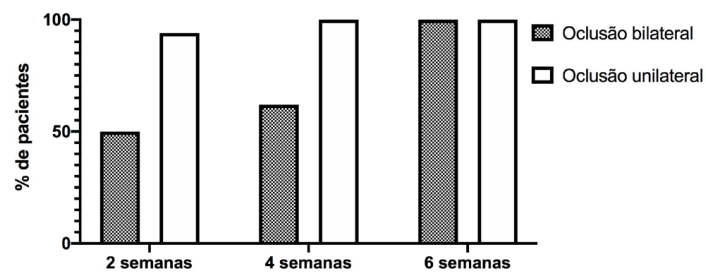

Figura 4. Porcentagem de pacientes com oclusão uni ou bilateral das veias safenas magnas em relação ao tempo

Tabela 1. Características individuais dos pacientes.

\begin{tabular}{lc}
\hline Idade (média, min-máx) & $50,4(35-68)$ \\
$\begin{array}{l}\text { Gênero masculino (n\%) } \\
\text { Diâmetro safena magna direita } \\
\text { (média } \pm \text { desvio padrão) }\end{array}$ & $16(29 \%)$ \\
$\begin{array}{l}\text { Diâmetro safena magna esquerda } \\
\text { (média } \pm \text { desvio padrão) }\end{array}$ & $6,31 \pm 1,23$ \\
\hline
\end{tabular}

Tabela 2. Classificação clínica, etiológica, anatômica e patológica (CEAP). C1 - telangiectasias ou veias reticulares; C2 - veias varicosas; C3 - edema; C4 - alterações cutâneas atribuídas a doença venosa; C5 - úlcera cicatrizada; C6 - úlcera ativa.

\begin{tabular}{ccc}
\hline Classificação CEAP & $\mathrm{n}$ & \multicolumn{1}{c}{$\%$} \\
\hline C1 & 0 & 0,00 \\
C2 & 7 & 13,00 \\
C3 & 10 & 18,00 \\
C4 & 22 & 40,00 \\
C5 & 5 & 9,00 \\
C6 & 11 & 20,00 \\
Total & 55 & 100,00 \\
\hline
\end{tabular}

Tabela 3. Número de punções para drenagem de coágulo retido em diferentes ocasiões e o número de pacientes em cada grupo.

\begin{tabular}{cc}
\hline $\begin{array}{c}\text { Número de punções com } \\
\text { drenagem de coágulo }\end{array}$ & Número de pacientes \\
\hline 0 & 36 \\
1 & 12 \\
2 & 7 \\
Total & 55 \\
\hline
\end{tabular}


Tabela 4. Classificação clínica, etiológica, anatômica e patológica (CEAP) em cada grupo conforme a necessidade de punção em diferentes ocasiões para drenagem de coágulo retido. Não houve diferença estatística entre os grupos.

\begin{tabular}{ccccccc}
\hline Classificação CEAP & 0 punção & $\%$ & 1 punção & \% & 2 punções \\
\hline C1 & 0 & 0,00 & 0 & 0,00 & 0 \\
C2 & 5 & 14,00 & 2 & 17,00 & 0,00 \\
C3 & 7 & 19,00 & 2 & 17,00 & 0,00 \\
C4 & 16 & 44,00 & 3 & 25,00 & 0,00 \\
C5 & 3 & 8,00 & 0 & 4,00 & 2 \\
C6 & 5 & 14,00 & 5 & 42,00 & 29,00 \\
Total & 36 & 100,00 & 12 & 100,00 & 7 \\
\hline
\end{tabular}

Comparando-se os pacientes nos quais houve oclusão bilateral das safenas e nos quais ocorreu oclusão unilateral na primeira sessão, não houve diferença significativa quanto à classificação CEAP e calibre das safenas e quanto à ocorrência de tromboflebite. Entre os pacientes que necessitaram de uma, duas ou três sessões para oclusão das duas veias safenas magnas, não houve diferença estatisticamente significativas quanto aos diâmetros venosos e quanto à classificação CEAP.

Foram observados 11 pacientes com classificação CEAP C6, com úlceras com diferentes graus de gravidade. $\mathrm{Na}$ revisão de 3 semanas, houve perda de seguimento de um paciente $(1,8 \%)$. Dos 10 pacientes restantes, $7(70 \%)$ estavam cicatrizados. Os outros $3(30 \%)$ estavam parcialmente cicatrizados, sendo que, em uma paciente que apresentava úlcera bilateral em um dos membros, houve inicialmente aumento do tamanho da úlcera.

\section{DISCUSSÃO}

A escleroterapia com espuma de polidocanol é um procedimento rápido, seguro e eficaz no tratamento das veias varicosas e da insuficiência venosa crônica dos membros inferiores. A grande maioria dos autores defende o seu uso através do tratamento unilateral. Os pacientes que apresentam sintomas graves bilateralmente são historicamente tratados um membro por vez ${ }^{12}$. Essa estratégia pode não ser adequada naqueles pacientes que necessitam de rápido tratamento, por exemplo, naqueles com feridas em ambos os membros inferiores. A escleroterapia com espuma de polidocanol em veias safenas magnas realizada bilateralmente e em tempo único é uma técnica ainda pouco estudada ${ }^{11}$.

A escleroterapia bilateral das veias safenas magnas mostrou-se eficaz, tanto do ponto de vista ultrassonográfico como do ponto de vista clínico (Figuras 5A e 5B). Observou-se, neste estudo, oclusão de $73,6 \%$ das safenas magnas com uma sessão; de 96,3\% com duas sessões e de $100 \%$ das safenas magnas em três sessões. Em 50\% dos pacientes, houve oclusão bilateral das veias safenas magnas em uma única sessão. Observaram-se resultados semelhantes em outros estudos. Bhogal, em 2010, descreveu 61 pacientes (122 membros) tratados

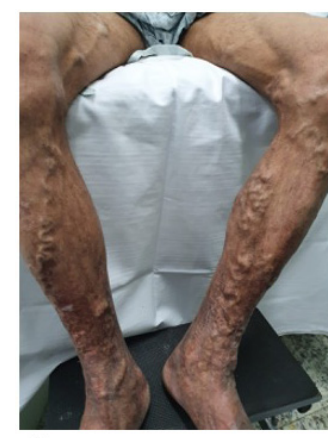

A

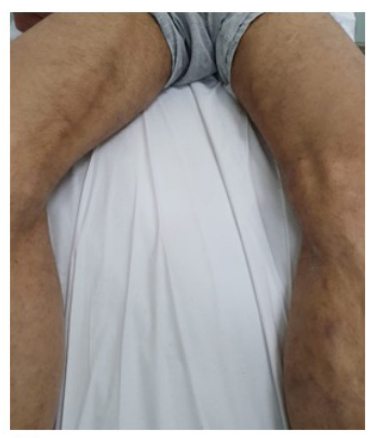

B
Figura 5. (A) Imagem antes e (B) 2 semanas após o procedimento. Este paciente foi submetido a uma sessão de escleroterapia com espuma de polidocanol a $3 \%$ em safenas magnas e a $1 \%$ em tributárias varicosas bilateralmente em tempo único com volume total de $20 \mathrm{~mL}$. Foi submetido também a uma sessão de punção e drenagem de coágulo retido.

por meio de escleroterapia com espuma de polidocanol em veias safenas magnas bilateralmente em tempo único e 51 pacientes (102 membros) tratados em dois tempos ${ }^{11}$. O autor baseou-se nos estudos de Rabe ${ }^{13}$ e no Consenso Australiano-Asiático de escleroterapia venosa guiada por ultrassonografia ${ }^{14}$ para definir o volume de espuma de polidocanol utilizado. Usando uma média de $17,3 \mathrm{~mL}$ de espuma para bilaterais em tempo único e $10 \mathrm{~mL}$ para cada tempo no grupo em dois tempos, $\mathrm{o}$ autor conseguiu um fechamento dos troncos safênicos em $81 \%$ e $70 \%$, respectivamente. Não houve aumento de complicações de um grupo em relação ao outro, com maiores taxas de oclusão do grupo tratado em tempo único. Dessa forma, os autores sugerem que o tratamento bilateral em tempo único é uma alternativa eficaz e segura em pacientes selecionados ${ }^{11}$.

Clinicamente, podemos inferir que a cicatrização completa $(70 \%)$ e parcial $(30 \%)$ das úlceras nos pacientes $\mathrm{C} 6$, em um tempo médio de 42 dias, indica uma melhora na qualidade de vida neste estudo. Por meio do Venous Clinical Severity Score (VCSS), avalia-se a gravidade da insuficiência venosa crônica através dos parâmetros numéricos, entre os quais a presença de úlceras ${ }^{15,16}$. Resultados semelhantes foram encontrados por Silva et al.: eles encontraram $89 \%$ de 
fechamento da úlcera em 791 dias em 19 pacientes submetidos a escleroterapia com espuma de polidocanol ${ }^{17}$. No estudo de Howard et al., foi observado um índice de cicatrização de úlcera de $86 \%$ em 12 meses $^{18}$. Coelho-Neto et al., em 2013, publicou um estudo em tratamento de pacientes com CEAP predominante avançada C5 e C6. A abordagem foi unilateral e usou dose máxima de $10 \mathrm{~mL}$ por sessão. Obteve $58 \%$ de sucesso em apenas em uma sessão. Houve melhora clínica estatisticamente significativa e cicatrização das úlceras em $85 \%$ dos pacientes em 30 dias $^{19}$.

Observa-se uma taxa de recanalização maior quando comparamos a escleroterapia com espuma à cirurgia convencional para tratamento de refluxo das veias safenas magnas. Figueiredo et al., em estudo randomizado pioneiro, encontraram uma taxa de oclusão de $90 \%$ na cirurgia aberta e de $78 \%$ na escleroterapia com espuma em 180 dias de seguimento ${ }^{20}$. Entretanto, mesmo nos pacientes com recanalização das veias safenas, observa-se uma redução do calibre venoso e redução da gravidade dos sintomas ${ }^{21}$.

Wright, em 2006, em estudo randomizado europeu comparando escleroterapia com espuma e cirurgia, reportou incidência de trombose venosa profunda em $5,3 \%$ dos pacientes ao injetar até $60 \mathrm{~mL}$ de espuma, o que levou o autor a reduzir o volume máximo para $30 \mathrm{~mL}$. Após a redução, 95 pacientes foram tratados sem novos episódios de trombose venosa profunda ${ }^{21}$.

Uma abordagem unilateral desde que limitada aos $10 \mathrm{~mL}$ preconizados pelas European Guidelines for Sclerotherapy in Chronic Venous Disorders é realizada sem controvérsias, pois o volume de espuma máximo recomendado é $10 \mathrm{~mL}$ por sessão nos casos de rotina ${ }^{12}$. O Consenso Australiano-Asiático de escleroterapia com espuma permite doses máximas maiores ${ }^{14}$. Neste estudo, utilizou-se volumes maiores de espuma baseados em trabalhos de autores os quais usaram volumes em torno de 20 mL ou até maiores em cada sessão. Khan Kharl et al., em 2019, no Paquistão, fizeram um estudo observacional prospectivo de 662 pacientes com 752 membros inferiores afetados por varizes, sendo $59 \%$ dos pacientes C2 e C3. Esses autores fixaram a dose máxima de espuma de polidocanol a 3\% em $20 \mathrm{~mL}$ e usaram a compressão inelástica. Embora 13,5\% tivessem comprometimento bilateral, todos foram tratados unilateralmente. O índice de oclusão das safenas foi de $67,5 \%$ com uma sessão, de $93,6 \%$ com duas sessões e de 99,4 em três sessões. A trombose venosa profunda ocorreu em três $(0,45 \%)$ pacientes, e dois $(0,3 \%)$ pacientes foram submetidos a flebectomia por tromboflebite superficial ${ }^{22}$. Bhogal et al. usaram uma mediana de $17,3 \mathrm{~mL}$ de espuma quando foi realizada escleroterapia bilateralmente em tempo único ${ }^{11}$. Wright usou um volume máximo de $30 \mathrm{~mL}$ de espuma quando comparou a escleroterapia com Varisolve ${ }^{\circledR}$ a cirurgia ou escleroterapia convencional ${ }^{23}$.

O benefício alcançado com a abordagem bilateral, embora elevando a dose de espuma acima da recomendada, é a oclusão das safenas em índices e sessões semelhantes aos da realizada para tratamento unilateral, aumentando o número de pacientes atendidos. Um dos inconvenientes apresentados é que a abordagem bilateral teoricamente aumenta o desconforto no pósprocedimento. Observou-se, neste estudo, uma alta taxa de coágulos retidos em 19 (34,5\%) pacientes. Resultados semelhantes foram encontrados por Ceratti et al. ${ }^{24}$, os quais encontraram $11 \%$, Nael e Rathbun ${ }^{25}, 17 \%$ e Kurnicki et al. ${ }^{26}, 21 \%$. Tremaine et al. ${ }^{27}$, ao avaliarem escleroterapia em membros superiores, encontraram uma taxa de $61,9 \%$ de coágulos retidos. Os pacientes os quais apresentaram coágulo retido foram submetidos a punção com agulha e drenagem do coágulo. Os pacientes os quais necessitaram de drenagem em mais de uma ocasião tendiam a ter insuficiência venosa crônica mais avançada.

Complicações mais graves, como trombose venosa profunda e alterações neurológicas permanentes, não foram observadas. Não houve trombose venosa profunda neste seguimento. Um paciente $(1,8 \%)$ apresentou lipotimia. Esses achados estão de acordo com a literatura. Ataques isquêmicos transitórios após escleroterapia com espuma foram diagnosticados em série com 1.025 pacientes $^{28}$, com melhora completa dos sintomas após 30 minutos. Acidente vascular encefálico foi descrito na literatura médica em três pacientes, todos com forame oval pérvio ${ }^{29}$.

Todos os pacientes deste estudo foram submetidos a enfaixamento compressivo dos membros com faixa inelástica em pós-operatório imediato. Além disso, foram orientados a usar meias elásticas em pós-operatório precoce. As indicações terapêuticas e profiláticas para meias elásticas, ataduras e compressões pneumáticas já estão bem estabelecidas. O International Compression $C l u b$ referendou essas evidências em reunião em Paris, em novembro de $2007^{30}$. Contudo, não há uma definição quanto à compressão pós-escleroterapia dos troncos safênicos. A diretriz recomenda que deve ser usada, e pode ser feita por meias elásticas ou bandagens ${ }^{26}$. Hamel-Desnos, em ensaio clínico randomizado em 60 pacientes com o uso de meias elásticas e sem nenhuma compressão no fechamento das safenas, não observou diferenças quanto à taxa de oclusão das veias safenas, às complicações e aos questionários de qualidade de vida ${ }^{31}$.

Além da compressão inelástica, das meias elásticas com pressões específicas ou não, há uma terceira opção, a compressão mista. Welsh, em 2017, em revisão sistemática, encontrou melhores resultados em termos de conforto, tolerabilidade e qualidade 
de vida na compressão mista quando comparada às compressões elástica e inelástica separadamente ${ }^{32}$.

Observam-se várias limitações neste estudo. Tratase de um estudo retrospectivo, com número reduzido de pacientes. Entretanto, é um tema ainda em aberto, com poucas publicações. Existe um hiato quando se compara o tratamento com escleroterapia com espuma bilateralmente em tempo único ao tratamento realizado em tempos distintos em pacientes com insuficiência venosa crônica bilateral. Este estudo pode fornecer dados para a prática clínica. Pacientes com doença venosa crônica avançada e bilateral e com difícil acesso aos sistemas de saúde podem se beneficiar da técnica descrita neste estudo. Há a necessidade de maiores estudos, com maior número de pacientes e, de preferência, prospectivos e aleatórios.

\section{CONCLUSÃO}

A escleroterapia com espuma de polidocanol em veias safenas magnas em tempo único mostrou-se técnica segura e eficaz em pacientes selecionados.

\section{REFERÊNCIAS}

1. Radhakrishnan N, Jayakrishnan R, Deepu G. Microfoam sclerotherapy for varicose veins: a retrospective analysis of a modified technique. Indian J Surg. 2015;77(Suppl 3):816-21. http://dx.doi.org/10.1007/ s12262-013-1013-2. PMid:27011463.

2. Kafejian O, Oliveira GA, Takayanagi T. Inovações técnicas na cirurgia de varizes visando a resultados estéticos. AMB Rev Assoc Med Bras. 1976;22(8):296-7. PMid:1086493.

3. Orbach EJ. Contributions to the therapy of the varicose complex. J Int Coll Surg. 1950;13(6):765-71. PMid:15428675.

4. Tessari L, Cavezzi A, Frullini A. Preliminary experience with a new sclerosing foam in the treatment of varicose veins. Dermatol Surg. 2001;27(1):58-60. PMid:11231246.

5. Frullini A, Cavezzi A. Sclerosing foam in the treatment of varicose veins and telangiectases: history and analysis of safety and complications. Dermatol Surg. 2002;28(1):11-5. http://dx.doi. org/10.1097/00042728-200201000-00003. PMid:11991262.

6. Bastos FR, Oliveira CCA, Paiva ACO. A história da escleroterapia. Rev Med Minas Gerais 2016.26:e1807. http://dx.doi. org/10.5935/2238-3182.20160107.

7. Evangelista SSM. Ecoescleroterapia com microespuma em varizes tronculares primárias. J Vasc Bras. 2006;5(3):167-8. http://dx.doi. org/10.1590/S1677-54492006000300002.

8. Bastos FR, de Lima AE, Assumpção AC. Ecoescleroterapia de varizes com espuma: revisão de literatura. Rev Assoc Med Minas Gerais. 2009; 19:38-43.

9. Hsu TS, Weiss RA. Foam sclerotherapy: a new era. Arch Dermatol. 2003;139(11):1494-6. PMid:14623712.

10. Couto-Junior EB. Abordagem não-paramétrica para cálculo do tamanho da amostra com base em questionários ou escalas de avaliação na área de saúde [tese]. São Paulo: Faculdade de Medicina, Universidade de São Paulo; 2009. http://dx.doi. org/10.11606/T.5.2009.tde-22022010-175431.
11. Bhogal RH, Moffat CE, Coney P, Nyamekye IK. Can foam sclerotherapy be used to safely treat bilateral varicose veins? Phlebology. 2012;27(1):1924. http://dx.doi.org/10.1258/phleb.2010.010027. PMid:21705479.

12. Rabe E, Breu FX, Cavezzi A, et al. European guidelines for sclerotherapy in chronic venous disorders. Phlebology. 2014;29(6):338-54. http:// dx.doi.org/10.1177/0268355513483280. PMid:23559590.

13. Rabe E, Pannier-Fischer F, Gerlach H, Breu FX, Guggenbichler S, Zabel M. Guidelines for sclerotherapy of varicose veins (ICD 10: 183.0, 183.1, 183.2, and 183.9). Dermatol Surg. 2004;30(5):687-93, discussion 693. PMid:15099309.

14. Myers K, Hannah P. Australasian college of phlebology. Directvision and ultrasound-guided sclerotherapy In: Myers K, Hannah P, Cremonese $M$, et al., editors. Manual of venous and lymphatic diseases. Boca Raton Florida: CRC Press; 2017. P. 137-147. http:// dx.doi.org/10.1201/9781315178288-12.

15. Lurie F, Passman M, Meisner M, et al. The 2020 update of the CEAP classification system and reporting standards. J Vasc Surg Venous Lymphat Disord. 2020;8(3):342-52. http://dx.doi.org/10.1016/j. jvsv.2019.12.075. PMid:32113854.

16. Rutherford RB, Padberg FT Jr, Comerota AJ, Kistner RL, Meissner $\mathrm{MH}$, Moneta GL. Venous severity scoring: An adjunct to venous outcome assessment. J Vasc Surg. 2000;31(6):1307-12. http:// dx.doi.org/10.1067/mva.2000.107094. PMid:10842165.

17. Silva MAM, Araujo ÁZP, Amaral JF, Jesus-Silva SG, Cardoso RS, Miranda FJr. Impacto da escleroterapia com espuma de polidocanol guiada por ultrassom em pacientes com úlcera venosa. J Vasc Bras. 2017;16(3):239-43. http://dx.doi.org/10.1590/1677-5449.002717. PMid:29930653.

18. Howard JK, Slim FJ, Wakely MC, et al. Recanalisation and ulcer recurrence rates following ultrasound-guided foam sclerotherapy. Phlebology. 2016;31(7):506-13. http://dx.doi.org/10.1177/0268355515598450. PMid:26224059.

19. Coelho F No, de Araújo GR, Kessler IM, de Amorim RFB, Falcão DP. Treatment of severe chronic venous insufficiency with ultrasound-guided foam sclerotherapy: a two-year series in a single center in Brazil. Phlebology. 2015;30(2):113-8. http://dx.doi. org/10.1177/0268355513517225. PMid:24335090.

20. Figueiredo M, Araújo S, Barros N Jr, Miranda FJr. Results of surgical treatment compared with ultrasound-guided foam sclerotherapy in patients with varicose veins: a prospective randomised study. Eur J Vasc Endovasc Surg. 2009;38(6):758-63. http://dx.doi. org/10.1016/j.ejvs.2009.07.015. PMid:19744867.

21. Oliveira RG, Morais D Fo, Engelhorn CA, Kessler IM, Coelho F No. Foam sclerotherapy for lower-limb varicose veins: impact on saphenous vein diameter. Radiol Bras. 2018;51(6):372-6. http:// dx.doi.org/10.1590/0100-3984.2017.0184. PMid:30559554.

22. Khan Kharl RA, Khan NI, Pervaiz HK, et al. Foam sclerotherapy: an emerging, minimally invasive and safe modality of treatment for varicose veins. J Ayub Med Coll Abbottabad. 2019;31(Suppl 1)(4):S641-5. PMID: 31965766

23. Wright D, Gobin JP, Bradbury AW, et al. Varisolve polidocanol microfoam compared with surgery or sclerotherapy in the management of varicose veins in the presence of trunk vein incompetence: European randomized controlled trial. Phlebology. 2006;21(4):180-90. http://dx.doi.org/10.1258/026835506779115807.

24. Ceratti S, Okano FM, Pontes AB, Pontes AL, Nastri R. Ecoescleroterapia com espuma no tratamento da insuficiência venosa crônica. Radiol Bras. 2011;44(3):167-71. http://dx.doi.org/10.1590/ S0100-39842011000300009.

25. Nael R, Rathbun S. Effectiveness of foam sclerotherapy for the treatment of varicose veins. Vasc Med. 2010;15(1):27-32. http:// dx.doi.org/10.1177/1358863X09106325. PMid:19797263. 
26. Kurnicki J, Osęka M, Tworus R, Gałązka Z. Ultrasound-guided foam sclerotherapy of great saphenous vein with $2 \%$ polidocanol - one-year follow-up results. Wideochir Inne Tech Malo Inwazyjne. 2016;11(2):67-75. http://dx.doi.org/10.5114/wiitm.2016.60579. PMid:27458485.

27. Tremaine AM, Friedmann DP, Goldman MP. Foam sclerotherapy for reticular veins of the dorsal hands: a retrospective review. Dermatol Surg. 2014;40(8):892-8. http://dx.doi.org/10.1097/ DSS.0000000000000076. PMid:25022711.

28. Gillet JL, Guedes JM, Guex JJ, et al. Side-effects and complications of foam sclerotherapy of the great and small saphenous veins: a controlled multicentre prospective study including 1025 patients. Phlebology. 2009;24(3):131-8. http://dx.doi.org/10.1258/ phleb.2008.008063. PMid:19470865.

29. Ma RWL, Pilotelle A, Paraskevas P, Parsi K. Three cases of stroke following peripheral venous interventions. Phlebology. 2011;26(7):280-4. http://dx.doi.org/10.1258/phleb.2010.010044. PMid:21422195.

30. Figueiredo $M$. A terapia da compressão e sua evidência científica.J Vasc Bras. 2009;8(2):100-2. http://dx.doi.org/10.1590/ S1677-54492009000200002.

31. Hamel-Desnos CM, Guias BJ, Desnos PR, Mesgard A. Foam sclerotherapy of the saphenous veins: randomised controlled trial with or without compression. Eur J Vasc Endovasc Surg. 2010;39(4):500-7. http:// dx.doi.org/10.1016/j.ejvs.2009.11.027. PMid:20097585.

32. Welsh L. What is the existing evidence supporting the efficacy of compression bandage systems containing both elastic and inelastic components (mixed-component systems)? A systematic review. J Clin Nurs. 2017;26(9-10):1189-203. http://dx.doi.org/10.1111/ jocn.13611. PMid:27706876.
Correspondência Luiz Antonio Miranda Rua dos Otoni, 909/2002, Bairro Santa Efigênia CEP: 30150-270 - Belo Horizonte (MG), Brasil

Tel.: +55 (31) 99997-2090 E-mail: luimir@hotmail.com

Informações sobre os autores LAM - Angiologista, Sócio efetivo, Sociedade Brasileira de Angiologia e Cirurgia Vascular (SBACV); Titular, Colégio Brasileiro de Cirurgiões

RCC - Cirurgiã vascular, Sócia aspirante, Sociedade Brasileira de Angiologia e Cirurgia Vascular (SBACV); Especialista em Ultrassonografia Vascular com Doppler, Associação Médica Brasileira (AMB), Colégio Brasileiro de Radiologia (CBR). CCSM - Angiologista, Cirurgiã vascular, Sócia efetiva, Sociedade Brasileira de Angiologia e Cirurgia Vascular (SBACV).

GCS - Cirurgião vascular, Sócio efetivo, Sociedade Brasileira de Angiologia e Cirurgia Vascular (SBACV); Professor Assistente, Departamento de Cirurgia, Faculdade de Medicina, Universidade Federal de Minas Gerais (UFMG), Mestrado e Doutorando em Cirurgia, UFMG.

Contribuição dos autores Concepção e desenho do estudo: LAM, GCS Análise e interpretação dos dados: LAM, CCSM, GCS Coleta de dados: LAM, RCC Redação do artigo: LAM, CCSM, GCS Revisão crítica do texto: LAM, CCSM, GCS Aprovação final do artigo*: LAM, RCC, CCSM, GCS Análise estatística: GCS Responsabilidade geral pelo estudo: LAM

*Todos os autores leram e aprovaram a versão final submetida ao J Vasc Bras. 